Research Article

\title{
Experimental Study on the Caustics of Moving Cracks and Elliptical Curvature under Impact Loading
}

\author{
Haohao Luo $\mathbb{D}^{1},{ }^{1}$ Renshu Yang, ${ }^{1,2}$ Yanbing Wang, ${ }^{1}$ Guoliang Yang, ${ }^{1}$ Chengxiao Li $\left(\mathbb{D},{ }^{1}\right.$ \\ Chen An, ${ }^{1}$ and Yuantong Zhang ${ }^{1}$ \\ ${ }^{1}$ School of Mechanics and Civil Engineering, China University of Mining and Technology (Beijing), Beijing 100083, China \\ ${ }^{2}$ School of Civil Engineering and Resources, University of Science and Technology, Beijing 100083, China \\ Correspondence should be addressed to Haohao Luo; 1964423767@qq.com
}

Received 14 January 2021; Revised 26 March 2021; Accepted 17 April 2021; Published 3 May 2021

Academic Editor: Francesco Colangelo

Copyright (C) 2021 Haohao Luo et al. This is an open access article distributed under the Creative Commons Attribution License, which permits unrestricted use, distribution, and reproduction in any medium, provided the original work is properly cited.

\begin{abstract}
A dynamic caustics test system was used, and different moving cracks were analysed to study the interaction between the crack growth rate, stress intensity factor, and curvature of the elliptical end of a moving crack under impact loading. Based on the linear elastic fracture mechanics theory, linearly fitting of the crack tip stress intensity factor and the elliptical curvature were employed to obtain the specific functional expressions. ABAQUS software was used to numerically simulate the moving crack fracture process passing through different elliptical curvatures. The crack tip stress intensity factor was calculated by the stress extrapolation method. The stress intensity factor obtained from the numerical calculation and the caustics test was consistent. The test and numerical simulation results showed that the direction of moving cracks entering and passing through the elliptical defects shows a certain regularity. As the ellipse curvature increased, the moving crack stress intensity factor passing through the ellipse gradually decreased, and the moving crack also passed easily through oval defects.
\end{abstract}

\section{Introduction}

During rock excavation, rock masses with joints, cracks, holes, and other defects are often encountered. Scholars at home and abroad have mostly researched joint angles, numbers, and directions, but there has been little research on interactions between moving cracks and crack tip curvature. Griffith suggested that there are many small cracks or defects in actual materials, and the sharp decline in macromaterial strength is mainly due to the large number of material defects. Therefore, it is necessary to study interactions between moving cracks and rock masses with defects.

Renshu Yang et al. [1] studied a polymethyl methacrylate (PMMA) directional fracture blasting test with defects and showed that, with increasing curvature on both sides of the specimen defect, the main crack peak propagation velocity and the peak dynamic stress intensity factor (SIF) gradually decreased. Liyun Yang et al. [2] conducted a caustic test on crack propagation evolution characteristics in the combined dynamic and static stress field and discussed the initial stress field effect on blasting crack propagation. Zhenhai Zhu [3] used a dynamic photoelastic test to observe the dynamic interaction between the explosion stress wave and the static radial crack produced by an explosion as well as the interaction between the reflected wave from the free boundary and the expanding crack. Yao et al. [4] used dynamic and static caustic methods in experimental research on plexiglass, aramid fibre composite material, and fibre-basic functionally graded material fractures with parallel cracks on the same and opposite sides under tensile stress waves. Camas et al. [5] carried out three-dimensional modelling of CT aluminium samples considering the three combinations of different load levels, specimen thicknesses, and crack end curvatures and showed that the plastic zone range depended on the applied load, the specimen thickness, and the crack curvature. When the curvature radius decreased, the plastic zone range expanded. Gao et al. [6] proposed a new theoretical model based on the crack tip plastic zone and shear zone (STZ) theory and studied the crack tip curvature radius evolution. Tomlinson and 
Patterson [7] studied reflection caustic images of precracked flat plates and precracked shell structures, thereby showing a clear curvature radius influence. Enling et al. [8] used a firstlevel light air gun loading system to analyse the CFRP laminate deformation process and failure mode with different curvatures. The principal stresses of each layer along the $0^{\circ}$ and $90^{\circ}$ laminate fibre directions were obtained. Zhong wen Yue et al. [9] studied the interaction between the propagation crack and cavity under the slotted hole explosive load. The results showed that the slotted hole and the burst crack directly penetrated the hole, and the hole had an obvious guiding effect with the burst crack appearing as a type I fracture. Qing Li et al. [10] studied the crack initiation time, crack growth rate, and fracture toughness of semidisc specimens with precracks of different inclination angles under impact loading. Zhong wen Yue et al. [11] studied the blast hole spacing effect on blasting crack growth in the cutting charge and showed that, with increased blast hole spacing, the burst cracks between two holes no longer penetrated directly. Rather, the deflection occurred in the "hand-in-hand" state, and the growth rate of burst cracks and peak dynamic stress intensity factor gradually decreased with increased blast hole distance. Jun Dai et al. [12] used numerical simulation methods to study the burst crack penetration mechanism between slits in rock and theoretically verified the slit pipe width feasibility. Guiyun Gao et al. [13] showed that, for specimens with the same compression deformation, the fracture toughness along the plastic compression direction was greater than the fracture toughness in the isotropic plane perpendicular to the plastic compression direction. As the compression deformation increased, the material fracture toughness was enhanced, and the brittle-toughness transition occurred. Wenfeng Hao et al. [14] conducted a dynamic caustics test using fibre bundles of different thicknesses and showed that the fibre bundle shielded the stress intensity factor and crack propagation speed at the crack tip. As the fibre bundle thickness increased, the shielding effect gradually increased. Xuefeng Yao et al. [15] studied the dynamic fracture behaviour of a three-point bending beam with offset cracks and analysed the influence of the initial crack distance from the beam centreline and half of the beam length on crack propagation behaviour. Shiwei Shen et al. [16] used dynamic caustics to study the double-hole rock mass blasting under different joint spacing conditions. The explosion stress wave propagated to the joints to deform the joint tip and guide the secondary cracks at the crack tip. Dongming Guo [17] used a digital laser caustics test system to study the blasting excavation influence on adjacent roadways. Compared with circular and arched roadways, the rectangular roadway damage was more direct from the direct blast side, which meant that the plane reflected more stress waves than the arc surface, resulting in stronger tensile failure. Yanbing Wang [18] used dynamic caustics to observe the cutting charge of different charge structures and used the finite element software LS-DYNA to calculate the relationship between the initial cutting charge blasting crack and the noncoupling coefficient. The results showed that the slit drug bag cutting effect with a noncoupling coefficient of 1.67 was the best.
Lang Lin et al. [19] showed that the crack growth rate, dynamic fracture toughness, and energy release rate all increased with increasing loading rate, and the delayed crack initiation time decreased with increasing loading rate. Jinjing Zuo explored the fracture dynamic properties of defect bodies under explosion loading by using the system of digital laser dynamic caustics; experimental results found that the fracture toughness increases with the decrease of the curvature [20]. Using the method that combines dynamic photoelasticity with dynamic caustics, Jing Fang studied the propagation mechanism of cracks under impact [21]. Chenxi Ding et al. found that, as the notch curvature decreases, both the peak value of the dynamic stress intensity factor and the corresponding peak value of the propagation velocity of the crack tip increase significantly after the initiation [22]. With the development of computer simulation technology, numerical simulation is an important method to solve the stress analysis in notched structure [23-25]. The authors in [26] studied the effects of U-shaped, $\mathrm{V}$-shaped, and I-shaped notches on the deformation mechanism of metal materials under impact loading by comprehensively using model experiments and numerical simulations.

Many scholars have studied the crack propagation path, crack propagation speed, and stress intensity factor at the crack tip through dynamic caustic tests. However, they seldom pay attention to the stress intensity factor and defect tip curvature when a moving crack passes through the flaw and cracks again. In this study, the dynamic caustics test system was used to study the prefabricated elliptical crack curvature influence on the stress intensity factor, propagation path, and propagation speed of the moving crack by changing the prefabricated ellipse tip curvature. The crack tip stress intensity factor was also calculated by stress extrapolation.

\section{Test Principle}

When stress in an engineering material changes, its optical properties also change. Due to the Poisson effect, the thickness of the solid decreases when subjected to tensile stress; the solid becomes a light-thin material with a corresponding decreased refractive index. After parallel light is reflected and refracted on the surface of a transparent object, bright and dark areas, called caustics, are formed on the reference plane.

Figure 1 illustrates the dynamic caustics test system used in this study. The system consisted of a laser source, a beam expander, a convex lens, a drop hammer, a high-speed camera, and a computer. The camera was a Fastcam-SA5, and the camera could take up to 75,000 pictures per second. The falling weight was $0.8 \mathrm{~kg}$, and the hammer drop height was $50 \mathrm{~cm}$. The consistent drop height of the hammer ensured that the crack growth in each impact test obtained the same energy.

The stress intensity factor is commonly used to characterize the crack tip stress field. In the caustics test system, the expression of the dynamic stress intensity factor and at the tip of the compound crack is in the following form [27]: 


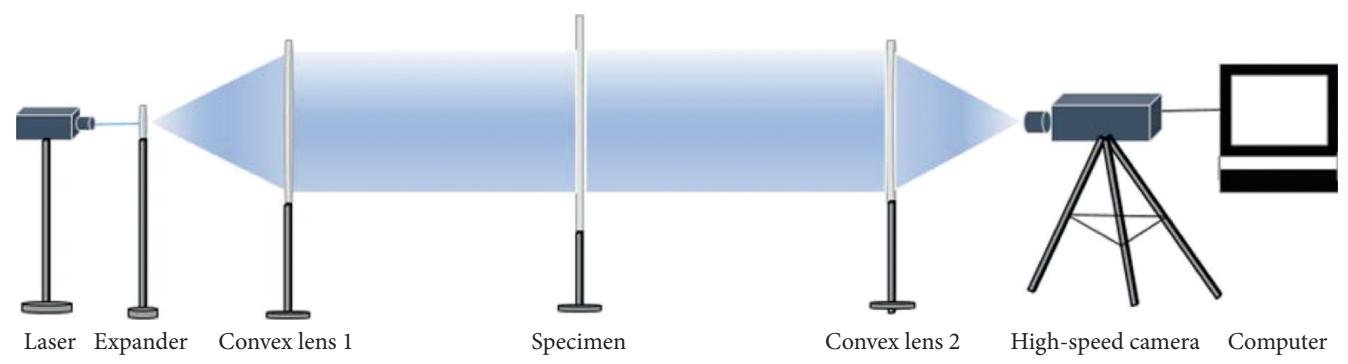

Figure 1: Digital laser dynamic caustics experimental system.

$$
\begin{aligned}
K_{I}^{d} & =\frac{2 \sqrt{2 \pi} F(v)}{3 g^{(5 / 2)} Z_{0} c d_{\mathrm{eff}}} D_{\max }^{(5 / 2)}, \\
K_{I I}^{d} & =\mu K_{I}^{d},
\end{aligned}
$$

where $D_{\max }$ is the maximum diameter of the focal speckle along the crack direction; $Z_{0}$ is the distance between the reference plane and the specimen plane; $c$ is the material optical stress constant; $d_{\text {eff }}$ is the specimen effective thickness; $\mu$ is the stress intensity factor proportional coefficient determined by the focal speckle maximum diameter $D_{\max }$ and minimum diameter $D_{\text {min }} ; g$ is the stress intensity factor related to the value of $\mu$; and $F(v)$ is the crack growth speed adjustment factor with a value of approximately 1 when the crack growth rate is lower than $0.6 c_{R}\left(c_{R}\right.$ is the Rayleigh wave velocity of the material).

Figure 2 shows the three-point bending elliptical crack type I fracture force model.

Assuming that the ellipse long axis is $2 \mathrm{a}$, the short axis is $2 \mathrm{~b}$, and the focal point coordinates are $(c, 0)$, the parametric equation of the plane ellipse is given in the following form:

$$
\begin{aligned}
& x=a \cos t \\
& y=b \sin t .
\end{aligned}
$$

The ellipse curvature expression can be calculated as follows [28]:

$$
\kappa(t)=\frac{a b}{\left(a^{2} \sin ^{2} t+b^{2} \cos ^{2} t\right)^{(3 / 2)}} .
$$

In the ellipse parametric equations $x=a \cos t$ and $y=b \sin t$, the coordinates at both ends are $(-a, 0)$ and $(a$, $0), t=0$ and $t=\pi$ are substituted into the ellipse curvature expression to obtain the curvature at both ends of the ellipse $\kappa(t)=\left(a / b^{2}\right)$, and the curvature radius expression at both ends of the ellipse is in the following form:

$$
\rho=\frac{1}{\kappa(t)}=\frac{b^{2}}{a} \text {. }
$$

The stress at the end of an elliptical crack is

$$
\sigma_{A}=\sigma\left(1+\frac{2 c}{a^{\prime}}\right)
$$

According to Inglis fracture theory [29], substituting the formula for the curvature radius into the above formula can be given:

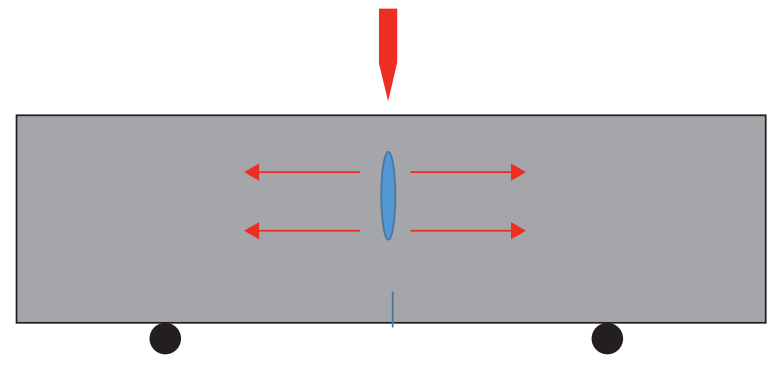

Figure 2: Schematic of the stress on the three-point bending elliptical crack type I fracture.

$$
\sigma_{A}=\sigma\left(1+2 \sqrt{\frac{c}{\rho}}\right)=\sigma(1+2 \sqrt{c \kappa(t)}),
$$

where $\sigma_{A}$ is the stress at the elliptical crack end, $\sigma$ is the applied stress, $a^{\prime}$ is the atomic distance, $\rho$ is the radius of curvature, and $2 c$ is the crack length.

Because $c \gg \rho$, then $(c / \rho)$ is large, omitting " 1 ." Then,

$$
\sigma_{A}=2 \sigma \sqrt{c \kappa(t)}
$$

According to the Griffith theory [29], $\sigma_{A}$ is equal to the theoretical bonding strength $\sigma_{\text {th }}$, and the crack is pulled apart to further expand, causing the material to break.

$$
\sigma_{\mathrm{th}}=\left(\frac{\gamma E}{a^{\prime}}\right)^{(1 / 2)} \text {. }
$$

The theoretical bonding strength $\sigma_{\text {th }}$ is only related to material constants such as the elastic modulus, surface energy, and atomic distance. Normally, the surface energy $\gamma$ is approximately $\left(a^{\prime} E / 100\right)$ [30]. Then,

$$
\sigma_{\text {th }}=\frac{E}{10} \text {. }
$$

The stress intensity factor (SIF) is a physical quantity that characterizes the stress field size at the crack tip and the relationship between the crack tip stress and the stress intensity factor. Based on linear elastic mechanics and brittle fracture theory, this study proposes the stress intensity factor influence coefficient $\eta$, namely, SIF $=\eta \sigma$. Peng Xu et al. [31] offered a similar discussion. When the distance between the measuring point and the crack tip approaches 0 , the stress intensity factor at the tip of a mode I crack has a linear relationship with the stress component perpendicular to the crack propagation direction. Let $\sigma_{A}=\sigma_{\text {th }}$ be the available applied stress $\sigma=\left(E / 20(c)^{(1 / 2)}\right)[\kappa(t)]^{-(1 / 2)}$. Then, the SIF can be obtained: 


$$
\mathrm{SIF}=\frac{\eta E}{20(c)^{(1 / 2)}}[\kappa(t)]^{-(1 / 2)} .
$$

Equation (10) shows that, as the curvature increases, the stress at the crack tip and the SIF decrease. Therefore, the stress at the crack tip is related not only to the elastic modulus but also to the crack tip curvature and increases and decreases accordingly.

\section{Test Plan}

PMMA was used as the test material. The basic physical and mechanical parameters included the longitudinal wave velocity $c_{\mathrm{p}}=2320 \mathrm{~m} / \mathrm{s}$, shear wave velocity $c_{\mathrm{s}}=1260 \mathrm{~m} / \mathrm{s}$, dynamic elastic modulus $E_{\mathrm{d}}=6.1 \mathrm{GPa}$, and Poisson's ratio $v$ $=0.31$. Plexiglass is a kind of rock-like material with good optical properties. The model size was $200 * 500 * 5 \mathrm{~mm}$, and a precrack with a length of $5 \mathrm{~mm}$ was opened at the bottom of the organic glass plate. For convenience, elliptical defects with curvatures of $20,5,1.25,0.56,0.31$, and $0.2 \mathrm{~mm}^{-1}$ were numbered L1 to L6 sequentially. The drop hammer weight was $0.8 \mathrm{~kg}$, the hammer drop height was $0.5 \mathrm{~m}$, and the drop hammer fell freely to the upper edge of the test piece. Each test ensured that the hammer drop height was the same so that the plexiglass obtained the same cracking energy. Table 1 lists the specimen dimensions.

\section{Results and Analysis}

Dynamic caustics were used to analyse the moving crack fracture process passing through the ellipse. The time was 0 when the drop hammer acted on the plexiglass. The caustic speckle shape was symmetrical along the crack propagation direction, which showed a type I crack. The focal speckle size indicated the degree of stress concentration at the crack tip.

When the hammer dropped, all six specimen types cracked from the centre, and the cracks began to expand from bottom to top. The position where the drop weight was applied was the centre of the specimen, which was a typical three-point bending fracture. The cracks were linear. When encountering prefabricated elliptical prefabricated defects with different curvatures, the cracks passed through the defects and continued to propagate forward until the specimen was completely broken. Without error, the falling weight loading position was the central specimen position, and the crack was subjected to tensile stress, which was a typical type I fracture. Excluding human factor influence and the specimen machining accuracy, the drop weight position may have been slightly left or right. The specimen was subjected to not only tensile stress but also in-plane shear stress. The specimen fracture was in the form of a I-II composite type fracture. Figure 3 shows the crack propagation trajectory of specimens L1-L6 under the drop hammer impact load. The cracks started to break from the lower part of the specimen, and the moving crack passed through the defect and continued to expand forward until the specimen was completely broken.

Figure 4 shows the dynamic focal speckle changes of a moving crack passing through an elliptical defect. When the drop hammer fell from a height of $50 \mathrm{~cm}$, the impact load acted on the test piece upper edge, and caustics appeared on the upper part of the elliptical prefabricated defect. The end of the linear prefabricated crack in the specimen centre also started to appear as a caustic spot. Unlike the focal speckle on the upper part of the linear precrack, the focal speckle radius on the upper part of the elliptical precrack first increased, then decreased, and then disappeared. The SIF between the elliptical defect and the upper specimen boundary did not exceed the plexiglass fracture toughness; there was stress concentration in this part, but no fracture. This may have been a direct effect of the falling weight with and part of the energy concentrated on the upper end of the elliptical prefabricated defect.

When stress concentrated at the linear precrack tip at the lower end of the specimen, the focal spot radius became larger. At that time, the linear precrack tip gathered energy, and the crack opened and expanded forward along the linear precrack, passing through the elliptical defect until the specimen was completely destroyed.

4.1. Crack Propagation Path Analysis. During the experiment, if the drop hammer position was slightly at the left or right of the centre, the moving crack direction entering the elliptical defect was different. If a moving crack entered an elliptical defect from the left side, the crack continued to expand forward along the middle and right sides of the elliptical defect. When a moving crack entered an elliptical defect from the right side, the crack continued to expand forward along the middle and left positions of the elliptical defect. When a moving crack entered an elliptical defect from the middle, the crack continued to expand forward along the left, middle, and right positions of the elliptical defect. We tested six groups of specimens, with each group containing three specimens for a total of 18 specimens. None of the specimens showed a moving crack entering from the left side of the defect and penetrating from the left side, nor entering from the right side of the defect and exiting from the right side. Figure 5 shows moving crack trajectories through elliptical defects.

4.2. Crack Movement Speed Analysis. The moving crack dynamic fracture process under impact loading with elliptical defects can be divided into four stages: I energy accumulation stage, II first crack, III through the hole, and IV secondary crack initiation.

Figure 6 shows the relationship curve between the crack growth rate and time under the L1 specimen impact load. I energy accumulation stage: as the drop hammer began to contact the test piece, the energy at the linear prefabricated crack tip began to accumulate. II first crack: at approximately $146.3 \mu \mathrm{s}$, the L1 specimen began to crack, and obvious cracks began to appear at the linear precrack tip. At that time, the crack propagation speed was $3.3 \mathrm{~m} / \mathrm{s}$. At $199.5 \mu \mathrm{s}$, the peak first cracking speed was $179.6 \mathrm{~m} / \mathrm{s}$. At approximately $266 \mu$ s, the moving crack expanded to an elliptical defect, and the crack propagation speed dropped rapidly to $76.4 \mathrm{~m} / \mathrm{s}$. III through the hole: during this process, 
TABLE 1: Specimen dimensions.

\begin{tabular}{lcccc}
\hline Number & Prefabricated defect shape & Ellipse curvature $\left(\mathrm{mm}^{-1}\right)$ & Long axis $(\mathrm{mm})$ & Short axis $(\mathrm{mm})$ \\
\hline L1 & Ellipse & 20 & 10 & 10 \\
L2 & Ellipse & 5 & 10 & 2 \\
L3 & Ellipse & 1.25 & 10 & 4 \\
L4 & Ellipse & 0.56 & 10 & 6 \\
L5 & Ellipse & 0.31 & 10 & 8 \\
L6 & Round & 0.2 & & 10 \\
\hline
\end{tabular}
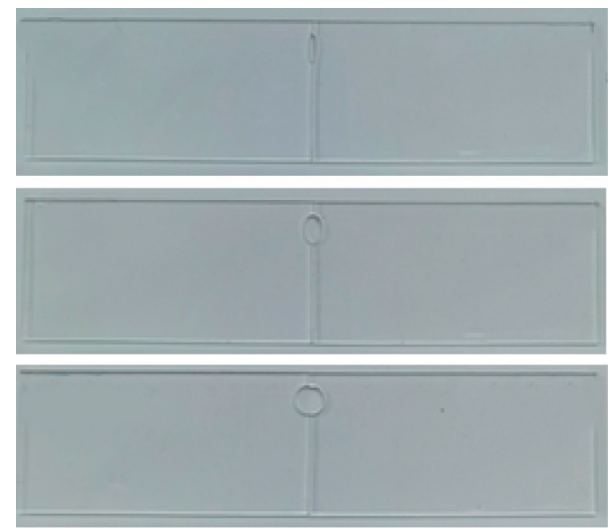

Figure 3: Specimen fracture schematic.

the crack growth rate dropped to zero rapidly, and energy at the upper end of the ellipse continued to gather. IV secondary crack initiation: at approximately $731.5 \mu \mathrm{s}$, the SIF at the end of the ellipse exceeded the material fracture toughness, and a secondary crack initiated from the upper end of the ellipse. At approximately $744.8 \mu \mathrm{s}$, the peak second crack initiation velocity was $192.1 \mathrm{~m} / \mathrm{s}$. Compared with the crack passing through the elliptical defect, the crack propagation speed increased after passing through the elliptical defect. This may have been because secondary cracking required more energy, and the crack tip energy had difficulty maintaining high-speed crack propagation, rapidly decreasing the crack propagation speed. The experimental data were consistent with the literature [32].

Due to space limitations, a description of the crack growth rate time history curves of specimens L1-L6 is not included. With the decreased elliptical end curvature, the peak secondary cracking occurred when the moving crack passed through the elliptical defect. The speed rose significantly, which may have been due to the elliptical end becoming increasingly dull, leading to elliptical end cracking requiring more energy to gather and a sharp crack propagation speed decline.

4.3. Dynamic Stress Intensity Factor Analysis. Figure 7 shows the L1 stress intensity factors over time. I energy accumulation stage: under the drop hammer impact load, the linear preformed crack tip focal speckle radius gradually became larger for the L1 specimen because the crack tip energy continued to accumulate. II first crack: cracking began at approximately $146.3 \mu \mathrm{s}$. The stress intensity factor at this time was $0.28 \mathrm{MPa} \cdot \mathrm{m}^{1 / 2}$. As the crack continued to grow forward, the stress intensity factor at the crack tip decreased slightly, and the stress intensity factor during crack propagation was lower than the stress intensity factor during crack initiation, indicating that the fracture toughness during crack propagation was less than the fracture toughness during crack initiation. III through the hole: as the moving crack coincided with the elliptical defect, the crack tip focal speckle temporarily disappeared, the stress intensity factor was 0 , and the focal speckle began to appear at the upper end of the elliptical defect where the energy continued to gather. IV secondary crack initiation: at approximately $731.5 \mu \mathrm{s}$, the crack started from the upper end of the ellipse for a second time, and at approximately $744.8 \mu$ s, the peak crack tip stress intensity factor was $0.31 \mathrm{MPa} \mathrm{m}^{1 / 2}$ when the stress intensity factor dropped rapidly until the test piece was broken.

The crack tip stress intensity factors in specimens L1-L6 were roughly similar. With the decreased elliptical curvature when the moving crack passed through the elliptical defect, the secondary cracking peak SIF also increased significantly.

4.4. Analysis of the Relationship between the Dynamic Fracture SIF and Curvature Change. Table 2 shows the peak stress intensity factors of the second crack initiation of the L1-L6 specimens passing through the elliptical defect. The second crack initiation peak stress intensity factor and the crack tip curvature expressions are given in (10), where SIF is the dynamic intensity factor; $\eta$ is the stress intensity factor influence coefficient; $2 \mathrm{c}$ is the prefabricated elliptical defect length; $E$ is the plexiglass elastic modulus; and $\kappa(t)$ is the elliptical prefabricated crack tip curvature.

Considering SIF from the above equation as the dependent variable $y, \kappa(t)$ is regarded as the independent variable $x$, and a new nonlinear analysis function in OriginPro8 software is obtained as $y=A^{*} x^{-(1 / 2)}$, where $y=$ $44.16 x^{-(1 / 2)}$ was obtained by the nonlinear fitting of the 6 sets of data, as shown in Figure 8. The correlation coefficient $R 2=0.996$ showed that these two variables had a strong correlation. From $A=\left(\eta E /\left(20(c)^{(1 / 2)}\right)\right)=44.16$, substituting $E=6.1 \mathrm{GPa}=6.1 \times 10^{3} \mathrm{MPa}$ and $c=5 \mathrm{~mm}=5 \times 10^{-3} \mathrm{~m}$, we obtain a value of $\eta=1.02 \times 10^{-2}$.

Substituting $\eta=1.02 \times 10-2$ into (10), we can obtain

$$
\mathrm{SIF}=5.12 \times 10^{-4} \frac{E}{(c)^{(1 / 2)}}[\kappa(t)]^{-(1 / 2)} .
$$

The crack tip curvature and length are reported based on the plexiglass three-point bending dynamic fracture caustic line test as well as the relationship between the crack tip SIF 


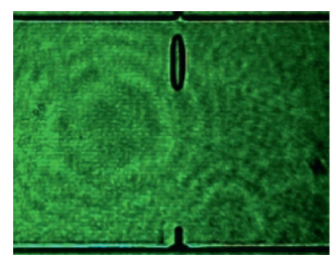

$\mathrm{t}=13.3 \mu \mathrm{s}$

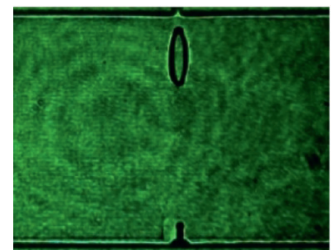

$\mathrm{t}=13.3 \mu \mathrm{s}$

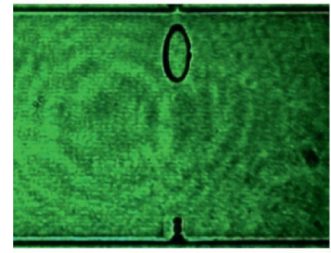

$\mathrm{t}=13.3 \mu \mathrm{s}$

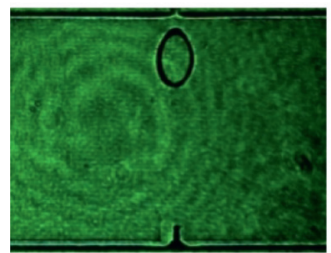

$\mathrm{t}=13.3 \mu \mathrm{s}$

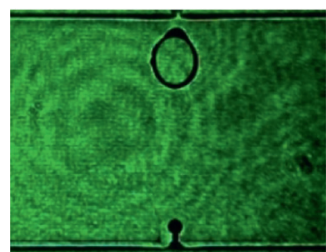

$\mathrm{t}=13.3 \mu \mathrm{s}$

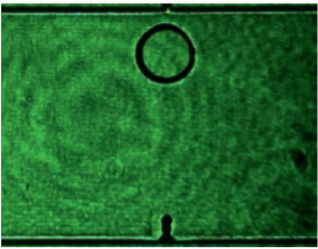

$\mathrm{t}=13.3 \mu \mathrm{s}$

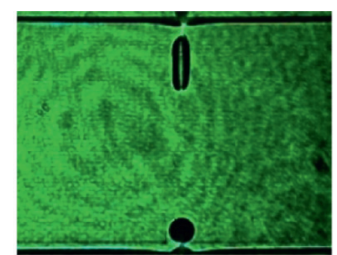

$\mathrm{t}=133 \mu \mathrm{s}$

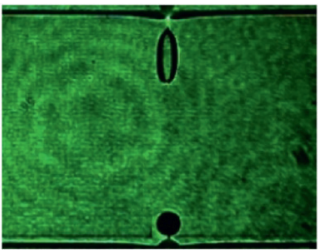

$\mathrm{t}=146.3 \mu \mathrm{s}$

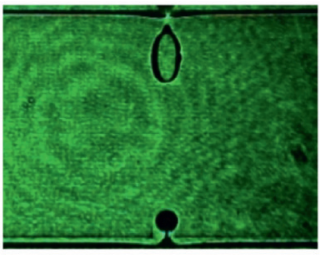

$\mathrm{t}=146.3 \mu \mathrm{s}$

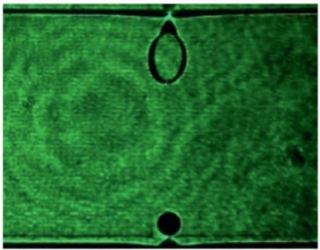

$\mathrm{t}=146.3 \mu \mathrm{s}$

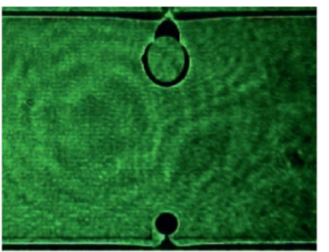

$\mathrm{t}=146.3 \mu \mathrm{s}$

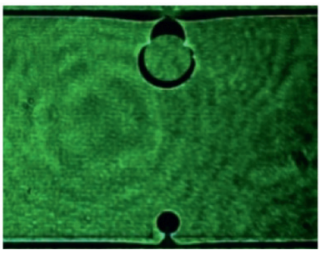

$\mathrm{t}=146.3 \mu \mathrm{s}$

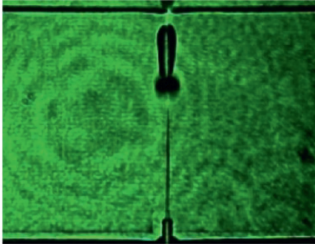

$\mathrm{t}=292.6 \mu \mathrm{s}$

(a)

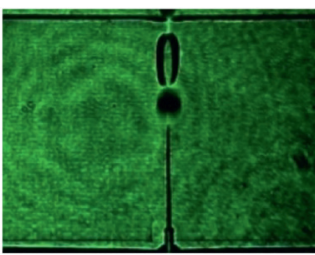

$\mathrm{t}=305.9 \mu \mathrm{s}$

(b)

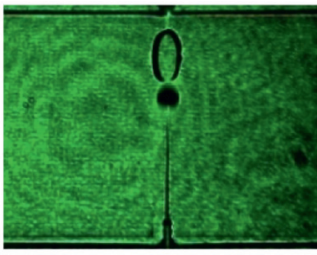

$\mathrm{t}=305.9 \mu \mathrm{s}$

(c)

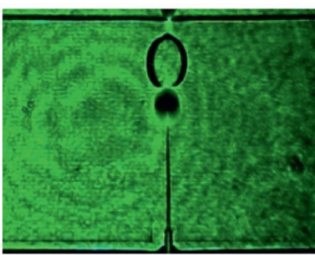

$\mathrm{t}=292.6 \mu \mathrm{s}$

(d)

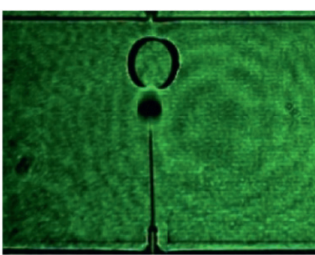

$\mathrm{t}=305.9 \mu \mathrm{s}$

(e)

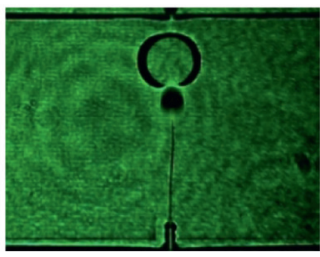

$\mathrm{t}=292.6 \mu \mathrm{s}$

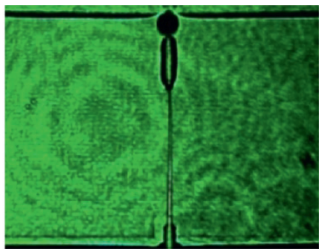

$\mathrm{t}=771.4 \mu \mathrm{s}$

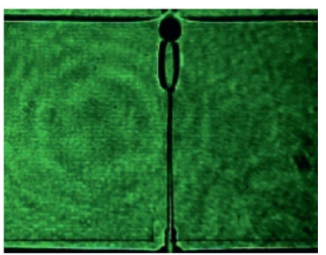

$\mathrm{t}=758.1 \mu \mathrm{s}$

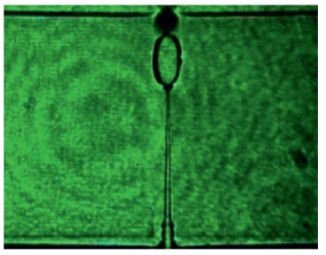

$\mathrm{t}=784.7 \mu \mathrm{s}$

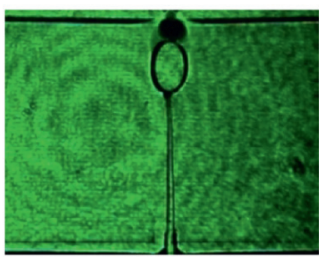

$\mathrm{t}=758.1 \mu \mathrm{s}$

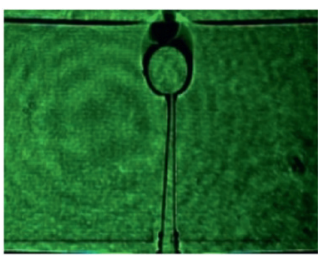

$\mathrm{t}=771.4 \mu \mathrm{s}$

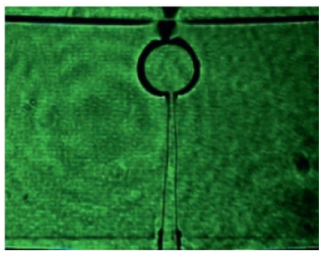

$\mathrm{t}=784.7 \mu \mathrm{s}$

(f)

Figure 4: Three-point curved focus speckle schematic. (a) L1. (b) L2. (c) L3. (d) L4. (e) L5. (f) L6. 


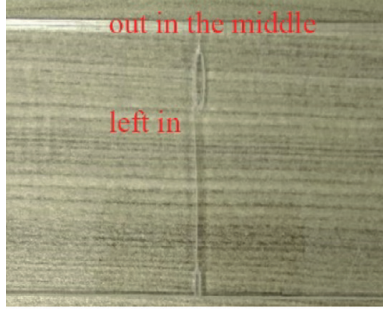

(a)

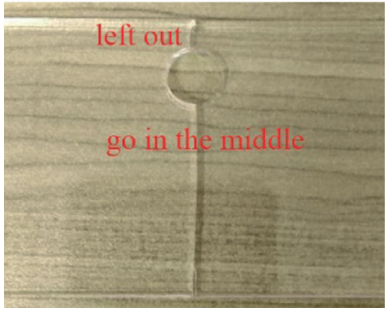

(e)

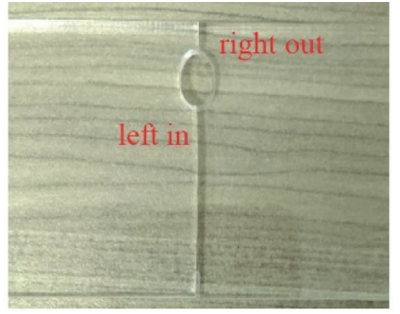

(b)

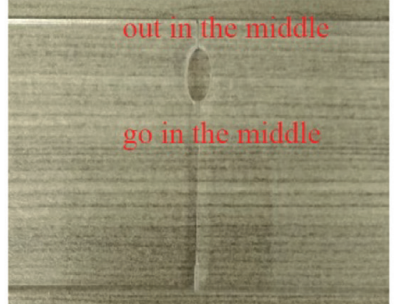

(f)

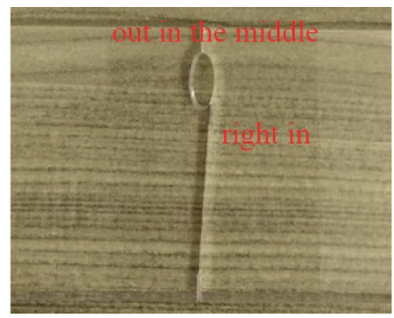

(c)

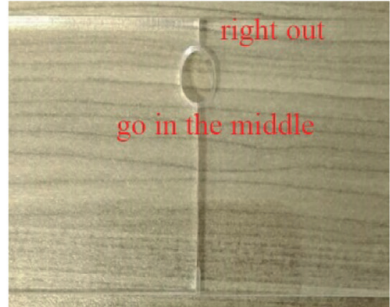

(g)

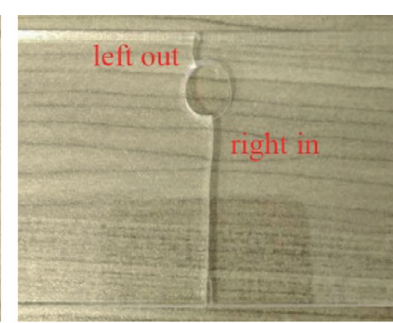

(d)

Figure 5: Moving crack trajectory diagrams through elliptical defects. (a) In from the left and out from the middle. (b) In from the left and out from the right. (c) In from the right and out from the middle. (d) In from the right and out from the left. (e) Into the middle and out from the left. (f) Into the middle and out from the middle. (g) Into the middle and out from the right.

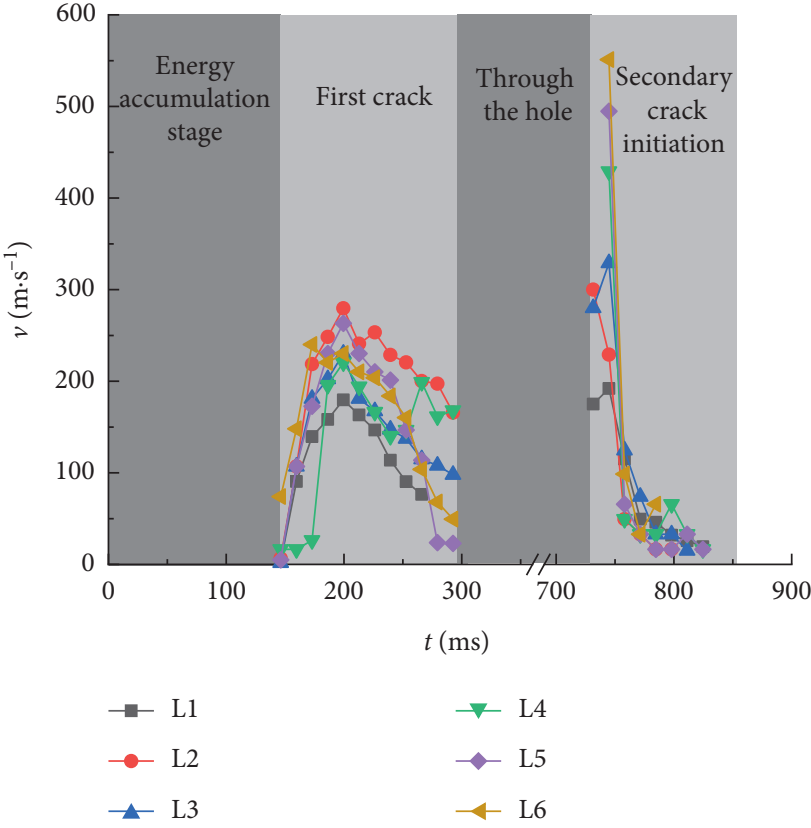

FIGURE 6: L1-L6 crack growth rate versus time curve.

and the material elastic modulus. Plexiglass is a homogeneous and isotropic brittle material, while rock is a nonhomogeneous and anisotropic material. Therefore, directly applying this equation may cause certain errors. However, the relationship between the crack tip curvature and fracture toughness is still a useful calculation for ideal states.

The degree of curvature of a smooth plane curve is defined as the derivative of the curvature radius; the greater the curvature, the greater the degree of curvature of the curve at that point. We also observe from the above equation

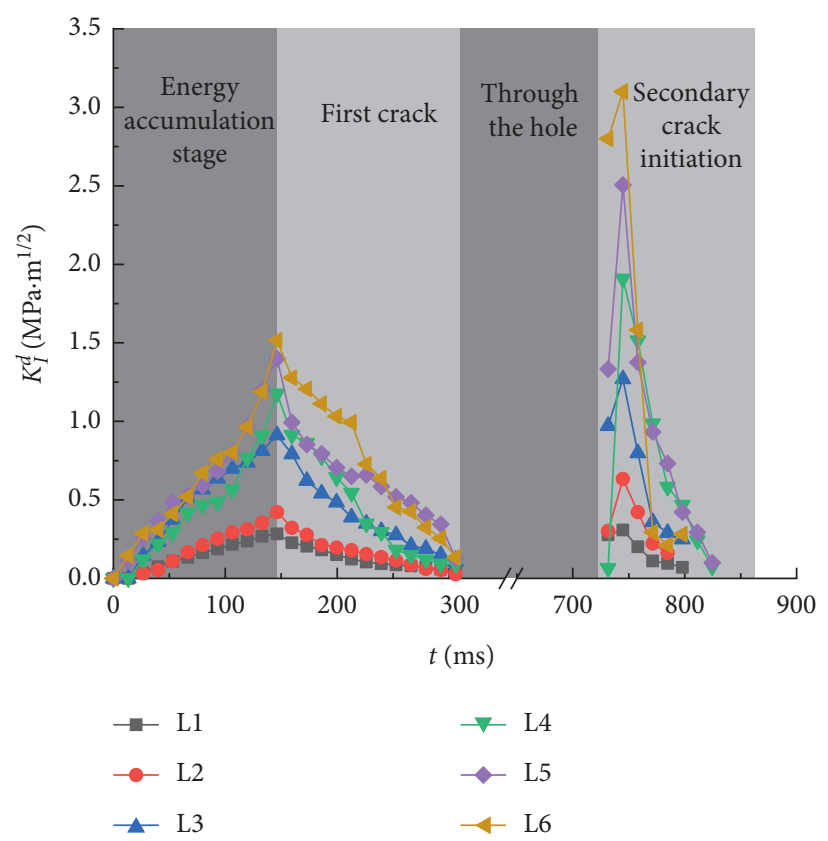

FIGURE 7: Stress intensity factor changes of specimens L1-L6 with time.

that, as the ellipse tip curvature increases, the SIF decreases sharply.

\section{Crack Growth Numerical Simulation}

This study used numerical simulation software ABAQUS to simulate the crack propagation process. The model size was the same as the above three-point bending test model at $200^{*} 50^{*} 5 \mathrm{~mm}$, and the material's elastic modulus, Poisson's 
TABLE 2: Crack tip curvature and stress intensity factor (SIF) parameters.

\begin{tabular}{lcc}
\hline Number & $\kappa(t) / \mathrm{m}-1$ & ${\mathrm{SIF} / \mathrm{MPa} \cdot \mathrm{m}^{1 / 2}\left(\mathrm{MN} \cdot \mathrm{m}^{1 / 2}\right)}$ \\
\hline L-1 & $20 \times 10^{3}$ & 0.31 \\
L-2 & $5 \times 10^{3}$ & 0.63 \\
L-3 & $1.25 \times 10^{3}$ & 1.27 \\
L-4 & $0.56 \times 10^{3}$ & 1.9 \\
L-5 & $0.31 \times 10^{3}$ & 2.5 \\
L-6 & $0.2 \times 10^{3}$ & 3.1 \\
\hline
\end{tabular}

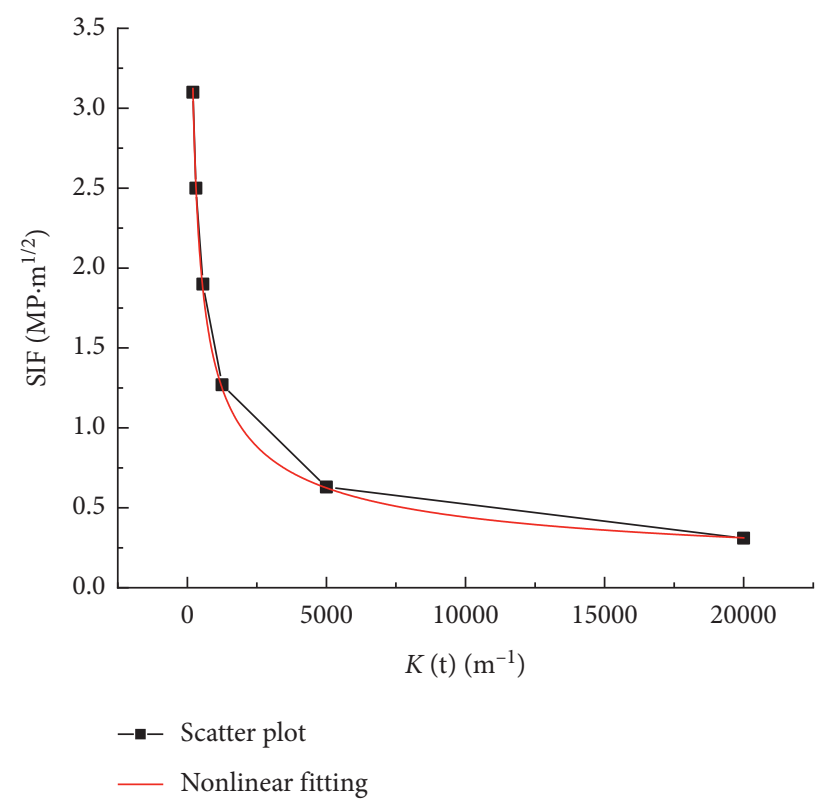

FIgURE 8: Crack initiation SIF with crack curvature.

ratio, and other mechanical parameters were also consistent with those above. The uniaxial compressive strength was $120 \mathrm{MPa}$, the falling weight height was $0.5 \mathrm{~m}$, the falling weight was $0.8 \mathrm{~kg}$, and the falling weight instantaneous velocity upon contact with the specimen was $3.13 \mathrm{~m} / \mathrm{s}$. The model used a two-dimensional variable shell element for simulation, and the model specimen used a quadrilateral plane stress element. The model had a total of 4,536 elements and 7,198 nodes. Crack initiation adopted the maximum principal stress failure criterion [33]. When the maximum principal stress in a certain direction of the element exceeded the tensile strength of time, the material failed and the crack started to grow. The model used a free boundary. Figure 9 shows the model mesh.

Figure 10 shows the von Mises stress cloud diagrams of the interaction process between the moving crack and the elliptical prefabricated defect under impact loading. When the falling hammer impact load acted on the specimen, stress concentration occurred at the contact point of the falling hammer and the test piece with two fulcrums at the upper end of the prefabricated linear crack. The von Mises stress cloud colour depth indicates the magnitude of the stress. The peak stress at the prefabricated crack tip at the bottom of the specimen increased with time. Before the crack initiated, the peak stress at the crack tip decreased, the stress peak oscillated slightly, and the crack began to crack. Before the moving crack passed through the elliptical prefabricated defect, the peak stress at the crack tip decreased, and the peak stress at the crack initiation point increased again compared with that before passing through the defect before finally breaking completely. Although only the L1 specimen stress cloud diagram is described here, specimens L2-L6 behaved similarly. The crack tip peak stress behaviour was consistent with the stress intensity factor in the caustics test.

Figure 11 gives the secondary cracking local stress cloud diagram when specimens L1-L6 passed through the elliptical defect. The stress attenuation was perpendicular to the crack propagation direction. With the decrease in the elliptical end curvature, secondary cracking occurred. The crack peak stress had a nonlinear correlation with the ellipse curvature. With decreasing curvature, the crack end peak stress decreased significantly.

A previous study [34] calculated the SIF at the tip of a mode I crack by extrapolating the element stress in the numerical calculation:

$$
\begin{aligned}
K_{I} & =\lim _{r \longrightarrow 0} \sqrt{2 \pi r_{i}} \sigma, \\
K_{I i} & =\sigma_{x i} \sqrt{2 \pi r_{i}},
\end{aligned}
$$

where $K_{I}$ is the stress intensity factor at the typeI crack tip, $r$ is the distance from the measuring point to the crack tip, and $\sigma_{x}$ is the stress component perpendicular to the crack propagation direction. 


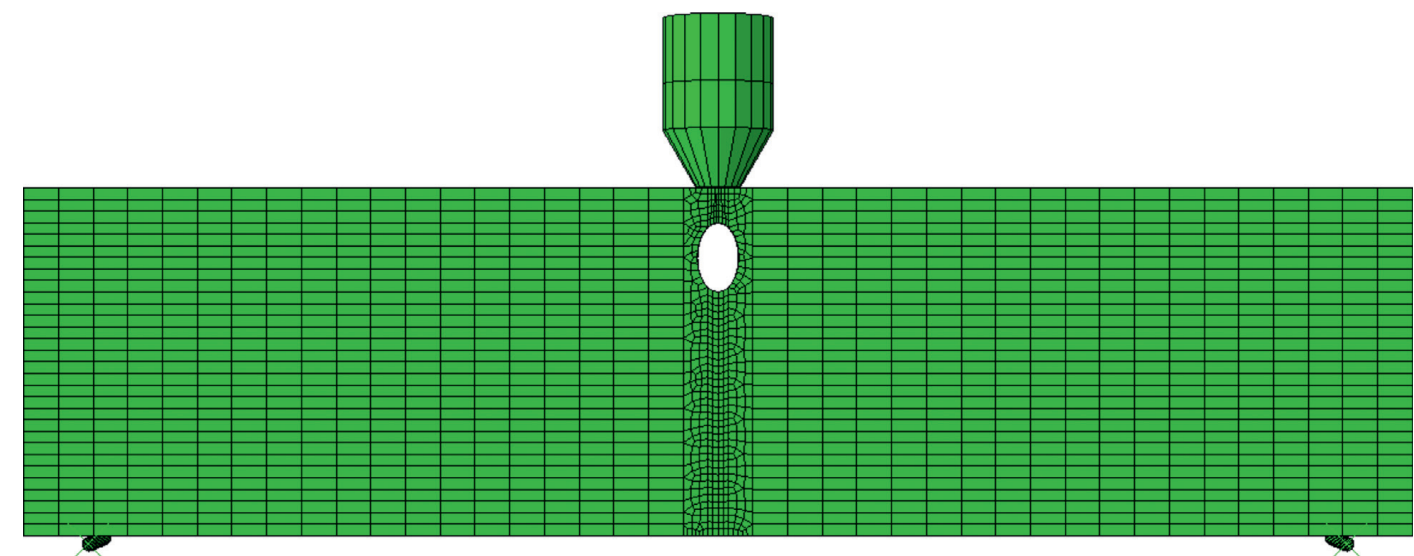

Figure 9: Model mesh schematic.

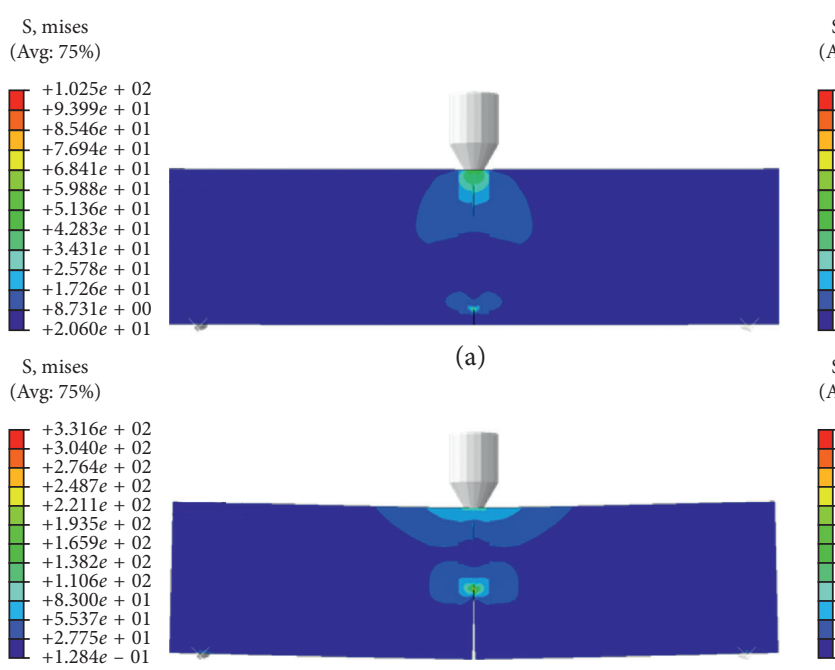

(c)

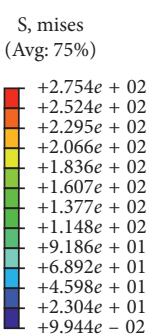

S, mises (Avg: 75\%)

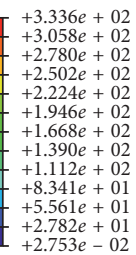

$+2.753 e-02$

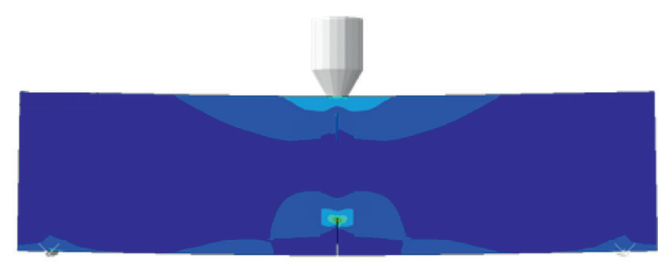

(b)

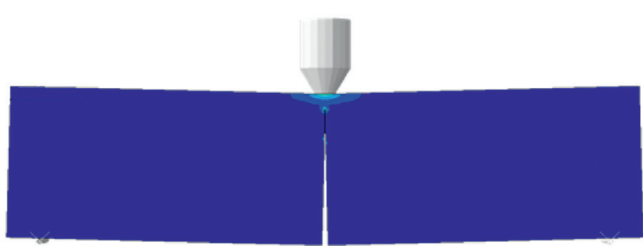

(d)

Figure 10: L1 specimen von Mise stress cloud diagram. (a) $76 \mu \mathrm{s}$. (b) $158 \mu \mathrm{s}$. (c) $302 \mu \mathrm{s}$. (d) $360 \mu \mathrm{s}$.

The least square method was used for linearly fitting $\left(r_{i}, K_{I i}\right)$. Assuming that the relationship between a and $b$ could be approximated by a linear relationship, then $\widehat{K}_{I}=A r+B$ when $r=0$ and $K_{I} \approx \widehat{K}_{I}(r=0)=B$.

Because the finite element software ABAQUS could not directly output the crack tip stress intensity factor, it was calculated indirectly by extracting the crack front element data.
A linear fitting was performed on $\left(r_{i}, K_{I i}\right)$ to obtain the crack tip stress intensity factor when L1-L6 cracked again, and the results were compared with the test results obtained from the caustics test, as shown in Figure 12. The error between the two results was acceptable. The discrepancy between the tip stress intensity factor when the crack started again and the literature $[5,6]$ may have been due to the defect boundary effect. 


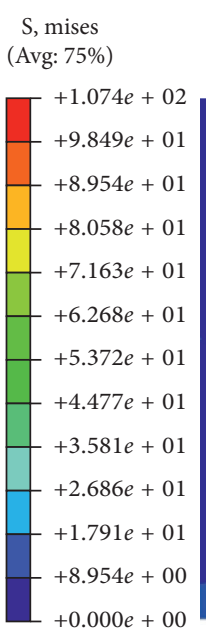

$\mathrm{S}$, mises (Avg: 75\%)

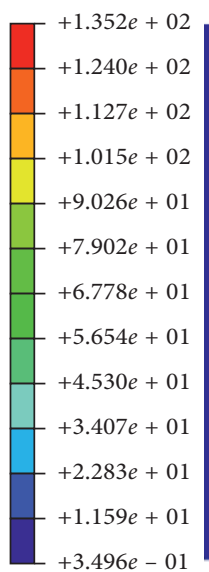

$+3.496 e-01$

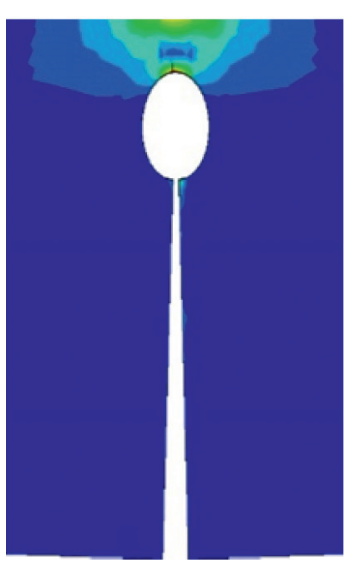

(d)

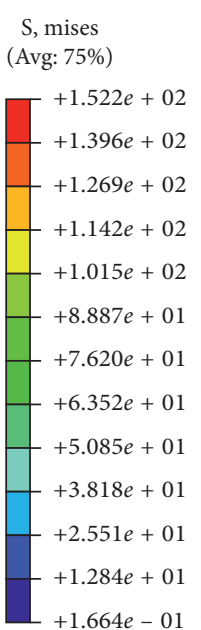

$\mathrm{S}$, mises (Avg: 75\%)

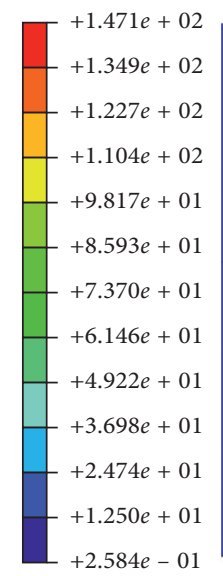

$+2.584 e-01$
S, mises

(Avg: 75\%)

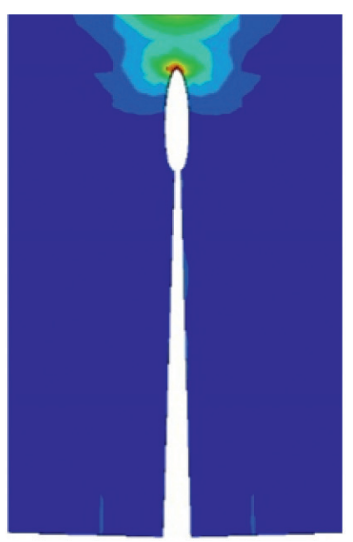

(b)

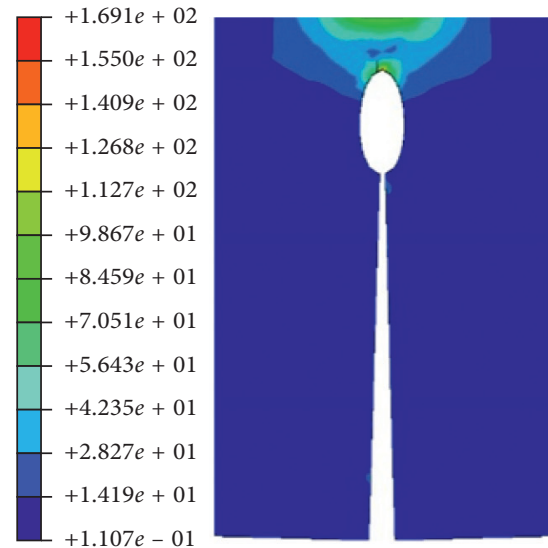

(c)

S, mises
$($ Avg. $75 \%)$

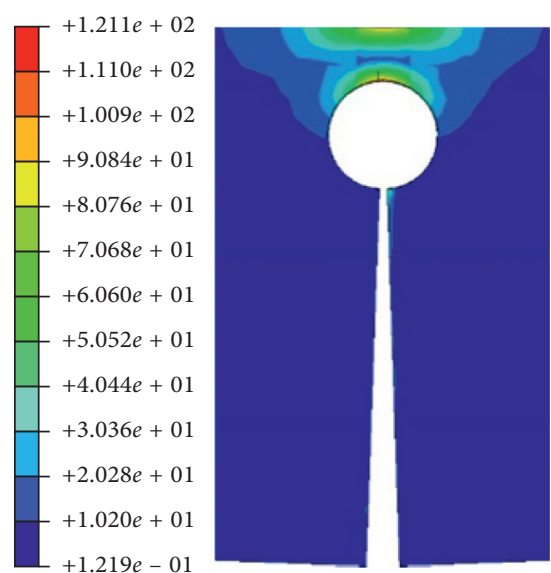

(f)

Figure 11: L1-L6 specimen von Mise stress cloud diagrams. (a) $\kappa(t)=20 \mathrm{~mm}^{-1}$. (b) $\kappa(t)=5 \mathrm{~mm}^{-1}$. (c) $\kappa(t)=1.25 \mathrm{~mm}^{-1}$. (d) $\kappa(t)$ $=0.56 \mathrm{~mm}^{-1}$. (e) $\kappa(t)=0.31 \mathrm{~mm}^{-1}$. (f) $\kappa(t)=0.2 \mathrm{~mm}^{-1}$.

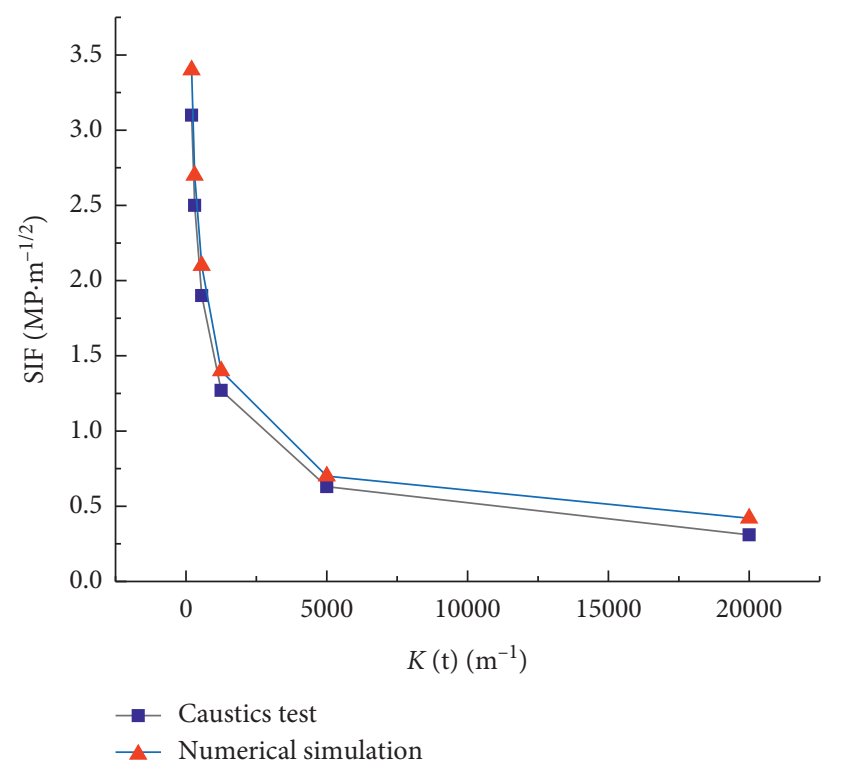

Figure 12: Comparison of the caustics test and numerical calculations. 


\section{Conclusions}

(1) When a moving crack encountered elliptical defects with different curvatures, the secondary crack initiation stress intensity factor changed. In this study, the influence coefficient of the stress intensity factor is proposed, and the expression of the secondary cracking stress intensity factor with the ellipse curvature of the ellipse is SIF $=2.14 \times 10^{-4}\left(E /(c)^{(1 / 2)}\right)$ $[\kappa(t)]^{-(1 / 2)}$. As the curvature increased, the stress intensity factor at the crack tip decreased. Under the same impact load, when the ellipse gradually changed from a circle to a line, it became easy for the moving crack to pass through the tip of the ellipse.

(2) When the drop hammer impacted the specimen, both the upper part of the elliptical defect and the upper end of the linear precrack produced focal speckles, and stress concentration occurred. The stress concentration in the upper part of the ellipse may have been due to the direct action of the falling weight, and part of the energy was concentrated at the upper end of the elliptical prefabricated defect. The focal speckles at the upper end of the linear and front cracks may have been due to the continuous accumulation of energy at the crack tip. The energy was concentrated to a certain extent, and the crack propagated forward along the linear preformed crack.

(3) After the moving crack entered the defect from the middle position of the lower defect tip, it expanded forward along the left, middle, and right three directions of the upper part of the defect. If the moving crack entered the defect from the left side of the lower defect tip, it expanded forward along the middle and right directions of the upper part of the defect. If the moving crack entered the defect from the right side of the lower defect tip, it expanded forward along the middle and left directions of the defect.

(4) Using the finite element software ABAQUS, the indirectly calculated stress intensity factor and the caustic test results were compared based on stress extrapolation. The error between the two was within an acceptable range.

\section{Data Availability}

All data can be obtained from the corresponding author upon request.

\section{Conflicts of Interest}

The authors declare that they have no conflicts of interest.

\section{References}

[1] R. S. Yang, C. X. Ding, L. Y. Yang et al., "Experimental study on controlled directional fracture blasting on PMMA mediums with flaws," Chinese Journal of Rock Mechanics and Engineering, vol. 36, no. 3, pp. 690-696, 2017.

[2] L. Yang, R. Yang, G. Qu, and Y. Zhang, "Caustic study on blast-induced wing crack behaviors in dynamic-static superimposed stress field," International Journal of Mining Science and Technology, vol. 24, no. 4, pp. 417-423, 2014.

[3] Z. H. Zhu, "Dynamic Photoelastic Test research on the effect of explosive stress wave on high speed propagating crack," Explosion and Shock Waves, vol. 2, pp. 175-185, 1993.

[4] X. F. Yao, W. Xu, S. L. Bai et al., "Caustics analysis of the crack initiation and propagation of graded materials," Composites Ence \& Technology, vol. 68, no. 3-4, pp. 953-962, 2008.

[5] D. Camas, J. Garcia-Manrique, and A. Gonzalez-Herrera, "Crack front curvature: influence and effects on the crack tip fields in bi-dimensional specimens," International Journal of Fatigue, vol. 44, no. 1, pp. 41-50, 2012.

[6] M. Gao, D. W. Ding, D. Q. Zhao, H. Y. Bai, and W. H. Wang, "Fracture morphology pattern transition dominated by the crack tip curvature radius in brittle metallic glasses," Materials Science and Engineering: A, vol. 617, pp. 89-96, 2014.

[7] R. A. Tomlinson and E. A. Patterson, "The effects of surface topography on the method of caustics," Experimental Mechanics, vol. 39, no. 4, pp. 335-342, 1999.

[8] E. L. Tang, H. T. Yin, X. Y. Gao et al., "Damage mechanism of CFRP laminates with different curvatures impacted by ice projectile at high velocity," Polymer Testing, vol. 93, 2021.

[9] Z. W. Yue, Y. Guo, and X. Wang, "Experimental study of crack propagation under blasting load in notched boreholes," Chinese Journal of Rock Mechanics and Engineering, vol. 34, no. 10, pp. 2018-2066, 2015.

[10] Q. Li, Y. Guo, and W. Q. Ma, "Dynamic caustics tests for semi-circular specimen under impact loading," Explosion and Shock Waves, vol. 35, no. 09, pp. 52-58, 2016.

[11] Z. W. Yue, S. Y. Tian, and Z. Y. Chen, "Influence of the interval between holes on crack propagation in slit charge blasting," Chinese Journal of Rock Mechanics and Engineering, vol. 37, no. 11, pp. 2460-2467, 2018.

[12] J. Dai, D. H. Wang, and G. H. Xiong, "Determination of slot width in a slotted pipe for directional-split blasting," Nonferrous Metals, vol. 04, pp. 110-113, 2004.

[13] G. Y. Gao, Z. Li, and M. Negahban, "Experimental study of the dynamic fracture behaviors of anisotropic brittle materials," Chinese Journal of Rock Mechanics and Engineering, vol. 38, no. S2, pp. 3979-3989, 2018.

[14] W. F. Hao, X. X. Sheng, G. P. Guo et al., "Mixed mode dynamic crack-fiber bundle interaction using caustics," Polymer Testing, vol. 55, pp. 13-26, 2016.

[15] X. F. Yao, C. Y. Xiong, and J. Fang, "Study of dynamic fracture behaviour onthree point bendbeamwith off centeredge crack," Chinese Journal of Theoretical and Applied Mechanics, vol. 06, pp. 22-30, 1996.

[16] S. W. Shen, W. W. Liao, Y. Xu et al., "Dynamic caustics test of rock mass under different joint spacing conditions with twohole blasting," Journal of China Coal Society, vol. 43, no. 08, pp. 2180-2086, 2018.

[17] D. Guo, B. Zhou, K. Liu, R. Yang, and P. Yan, "Dynamic caustics test of blast load impact on neighboring different cross-section roadways," International Journal of Mining Science and Technology, vol. 26, no. 5, pp. 803-808, 2016.

[18] Y. Wang, "Study of the dynamic fracture effect using slotted cartridge decoupling charge blasting," International Journal of Rock Mechanics and Mining Sciences, vol. 96, pp. 34-46, 2017.

[19] L. Lang, Z.-m. Zhu, H.-b. Wang, J.-w. Huang, M. Wang, and X.-s. Zhang, "Effect of loading rates on crack propagating 
speed, fracture toughness and energy release rate using singlecleavage trapezoidal open specimen under impact loads," Journal of Central South University, vol. 27, no. 8, pp. 24402454, 2020.

[20] R. S. Yang and J. J. Zuo, "Experimental research on explosively driven crack initiation and propagation from flaws of various geometry," Journal of Testing and Evaluation, vol. 48, no. 02, pp. 1138-1154, 2020.

[21] J. Fang, X. F. Yao, and C. Y. Xiong, "Experimental study on crack curving propagation in bending beams under impulsive load," Acta Mechanica Sinica, vol. 16, no. 01, pp. 83-92, 2000.

[22] C. X. Ding, R. S. Yang, C. L. Xiao et al., "Influence of the notch curvature on the impact fracture of the three-point bending beam," Engineering Fracture Mechanics, vol. 241, no. 01, pp. 23-31, 2021.

[23] S. H Ju, "A hybrid analytical-numerical solution for 3D notched plates," International Journal of Solids and Structures, vol. 51, no. 23, pp. 1138-1154, 2014.

[24] A. Ince, "Numerical validation of computational stress and strain analysis model for notched components subject to nonproportional loadings," Theoretical and Applied Fracture Mechanics, vol. 84, no. 01, pp. 26-37, 2016.

[25] Y. Mikami, T. Kawabata, T. Tagawa et al., "Numerical simulation of residual stress modification by reverse bending of notched fracture toughness test specimens of multipass welds," Theoretical and Applied Fracture Mechanics, vol. 92, no. 01, pp. 214-222, 2017.

[26] S. V. Panin, P. O. Maruschak, I. V. Vlasov et al., "The role of notch tip shape and radius on deformation mechanisms of $12 \mathrm{Cr} 1 \mathrm{MoV}$ steel under impact loading. Part 1. Energy parameters of fracture," Fatigue \& Fracture of Engineering Materials \& Structures, vol. 40, no. 4, pp. 586-596, 2017.

[27] R. S. Yang and L. B. Gui, Caustics Method and its Application, China University of Mining and Technology Press, Xuzhou, China, 1997.

[28] S. L. Xu, Y. D. Jin, Z. S. Hu et al., Differential Geometry Learning Guide, University of Science and Technology of China Press, Hefei, China, 2014.

[29] W. G. Wang, Advanced Rock Mechanics Theory, Metallurgical Industry Press, Beijing, China, 1996.

[30] Z. D. Guan and Z. T. Zhang, Physical Properties of Inorganic Materials, Tsinghua University Press, Beijing, China, 2011.

[31] R. S. Yang, P. Xu, and Z. W. Yue, "Dynamic fracture analysis of crack-defect interaction for mode I running crack using digital dynamic caustics method," Engineering Fracture Mechanics, vol. 161, no. 03, pp. 243-250, 2017.

[32] Q. Li, H. J. Cao, M. Z. Yang et al., "Experimental investigation on dynamic fracture toughness of offset crack material using caustic method," Journal of Mining Science and Technology, vol. 2, no. 03, pp. 243-250, 2017.

[33] S. Deng, Z. M. Zhu, L. Wang et al., "Study on the influence of in-situ stresses on dynamic fracture behaviors of cracks," Chinese Journal of Rock Mechanics and Engineering, vol. 38, no. 10, pp. 1989-1999, 2019.

[34] D. Xie, Q. Qian, and C. A. Li, Numerical Calculation Methods and Engineering Applications in Fracture Mechanics, Science Press, Beijing, China, 2009. 